\title{
X-ray emission from MP Muscae: an old classical T Tauri star
}

\author{
C. Argiroffi ${ }^{1}$, A. Maggio $^{2}$, and G. Peres ${ }^{1}$ \\ 1 Dipartimento di Scienze Fisiche ed Astronomiche, Sezione di Astronomia, Università di Palermo, Piazza del Parlamento 1, \\ 90134 Palermo, Italy \\ e-mail: [argi; peres] @astropa.unipa.it \\ 2 INAF - Osservatorio Astronomico di Palermo, Piazza del Parlamento 1, 90134 Palermo, Italy \\ e-mail: maggio@astropa.unipa.it
}

Received 22 December 2006 / Accepted 25 January 2007

ABSTRACT

\begin{abstract}
Aims. We study the properties of X-ray emitting plasma of MP Mus, an old classical T Tauri star. We check whether an accretion process could produce the observed X-ray emission and we derive the accretion parameters and the characteristics of the shockheated plasma. We compare the properties of MP Mus with those of younger classical T Tauri stars to test whether age is related to the properties of the $\mathrm{X}$-ray emitting plasma.

Methods. XMM-Newton X-ray spectra allow us to measure plasma temperatures, abundances, and electron density. The density of cool plasma probes whether X-ray emission is produced by plasma heated in the accretion process.

Results. X-ray emission from MP Mus originates from high density cool plasma but a hot flaring component is also present, suggesting that both coronal magnetic activity and accretion contribute to the observed X-ray emission. We find a Ne/O ratio similar to that observed in the much younger classical T Tauri star BP Tau. From the soft part of the X-ray emission, mostly produced by plasma heated in the accretion shock, we derive a mass accretion rate of $5 \times 10^{-11} M_{\odot} \mathrm{yr}^{-1}$.
\end{abstract}

Key words. stars: abundances - stars: circumstellar matter - stars: coronae - stars: individual: MP Muscae stars: pre-main sequence $-\mathrm{X}$-rays: stars

\section{Introduction}

Low mass stars are sources of strong X-ray radiation, starting in their early evolutionary phases. Coronal plasma, responsible for the X-ray emission, is confined and probably heated by magnetic fields that emerge from the stellar surface (Feigelson \& Montmerle 1999; Preibisch et al. 2005). The coronal plasma observed in essentially all late-type stars can be characterized by a large variety of average temperatures (from a few MK to tens of MK) and metallicities (from one tenth to a few times the solar photospheric value), but a common feature is the low density measured at the temperature of formation of $\mathrm{O}$ VII He-like triplets $\left(N_{\mathrm{e}} \approx 10^{10}-10^{11} \mathrm{~cm}^{-3}\right.$ at $\left.T \sim 2 \mathrm{MK}\right)$ (Testa et al. 2004; Ness et al. 2004).

In very young stars, however, accretion may cause X-ray emission in addition to a magnetically-confined coronal plasma. In classical T Tauri stars (CTTSs), gas falls from the circumstellar envelope, funnelled by the magnetic field, and hits the stellar photosphere. In the resulting shock the accreted material is heated to temperatures of a few MK (Calvet \& Gullbring 1998). With typical mass accretion rates, the shock-heated plasma can reach X-ray luminosities as high as $10^{31} \mathrm{erg} \mathrm{s}^{-1}$.

The few CTTSs for which high-resolution X-ray spectroscopy has been performed show, in most cases, cool plasma components $(2-4 \mathrm{MK})$ with high electron densities $\left(10^{11}-10^{13} \mathrm{~cm}^{-3}\right)$ which have been interpreted as evidence for $\mathrm{X}$-ray emission due to an accretion shock. The best known examples of this behavior are the CTTSs TW Hya, BP Tau, and
V4046 Sgr (Kastner et al. 2002; Schmitt et al. 2005; Günther et al. 2006). Noticeable exceptions are the CTTS T Tau (Güdel et al. 2007) and the Herbig star AB Aur (Telleschi et al. 2007).

In the hypothesis of X-ray emission originating in shocks, attention has been focused also on plasma element abundances. They probe the chemical composition of the accreting stream, and hence provide indications of the physical and chemical processes at work in the inner circumstellar disk (Stelzer \& Schmitt 2004; Drake et al. 2005).

In this letter we present an XMM-Newton observation of MP Muscae, one of the oldest known CTTSs, that aimed to study the properties of the X-ray emitting plasma and the role of the accretion process. MP Mus is a K1 IVe star of the Lower Centaurus Crux (LCC) association. Gregorio-Hetem et al. (1992) first identified it as a CTTS by measuring enhanced $\mathrm{H} \alpha$ emission $(E W=$ $-47 \AA)$ and $\mathrm{Li}$ absorption $(E W=0.37 \AA)$. Excesses in infrared bands revealed an optically thick circumstellar disk (Mamajek et al. 2002; Silverstone et al. 2006) with an estimated dust mass of $\sim 5 \times 10^{-5} M_{\odot}$ (Carpenter et al. 2005). Batalha et al. (1998) derived a rotational period of $5.75 \mathrm{~d}$ from variability in the $B, V, R$, and $I$ bands, although the amplitude of variability is surprisingly low for a CTTS.

Mamajek et al. (2002) derived three different ages for MP Mus, 7, 14, and 17 Myr, depending on the adopted theoretical evolutionary tracks. The LCC association, to which MP Mus belongs, is the oldest portion of the Scorpius-Centaurus $\mathrm{OB}$ association, and its estimated age is between 16 and $23 \mathrm{Myr}$ (Mamajek et al. 2002; Sartori et al. 2003). 


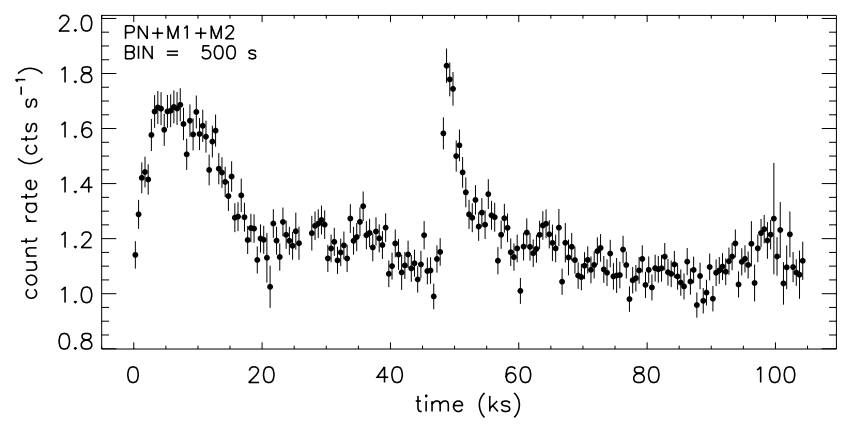

Fig. 1. Background-subtracted light curve of MP Mus obtained by adding the three EPIC instrument data.

Table 1. MP Mus best-fit paramenters.

\begin{tabular}{lccc}
\hline \hline Par. & \multicolumn{2}{c}{ best-fit value } \\
\hline$T^{a}$ & $2.7_{-0.2}^{+0.1}$ & $7.2_{-0.5}^{+0.4}$ & $36_{-11 .}^{+18}$ \\
$\mathrm{EM}^{b}$ & $9.6_{-2.5}^{+5.2}$ & $17.9_{-3.0}^{+4.0}$ & $2.9_{-0.8}^{+1.5}$ \\
$N_{\mathrm{H}}^{c}$ & $4.6_{-1.7}^{+1.8}$ & & \\
$\mathrm{Ab}^{d}{ }^{d}$ & $\mathrm{O}=0.25_{-0.07}^{+0.08}$ & $\mathrm{Ne}=0.76_{-0.15}^{+0.23}$ & $\mathrm{~S}=0.28 \pm 0.20 \mathrm{Fe}=0.09_{-0.02}^{+0.04}$ \\
\hline
\end{tabular}

${ }^{a}$ Temperature (MK). ${ }^{b}$ Emission Measure $\left(10^{52} \mathrm{~cm}^{-3}\right) .{ }^{c}$ Hydrogen column density $\left(10^{20} \mathrm{~cm}^{-2}\right){ }^{d}$ Abundances refer to the solar photospheric values of Asplund et al. (2005). All the uncertainties correspond to the $68 \%$ confidence level.

\section{Observation and data analysis}

MP Mus was observed with XMM-Newton for a duration of $\sim 110 \mathrm{ks}$ on 2006 August 19-20. We processed the data using the SAS V6.5 standard tasks. After having discarded time segments affected by high background count rates, we obtained good time intervals adding up to $\sim 100 \mathrm{ks}$. The X-ray light curve (Fig. 1) shows clear hints of flaring activity, typical of magnetically active coronal sources.

To increase the signal-to-noise ratio we rebinned the PN and MOS spectra to obtain at least 30 counts in each bin, and the RGS wavelength bins were joined two by two. Spectral analysis was performed using the Astrophysical Plasma Emission Database (APED V1.3, Smith et al. 2001). We adopted the Asplund et al. (2005) solar photospheric composition as the reference set of element abundances.

We derived the characteristics of the X-ray emitting plasma of MP Mus by fitting PN and MOS spectra with XSPEC V11.3.0 (Arnaud 1996), adopting an optically-thin plasma emission model with three isothermal components. The best-fit 3-T model (Table 1) also provided individual $\mathrm{O}, \mathrm{Ne}, \mathrm{Fe}$, and $\mathrm{S}$ abundances, while the abundances of the other elements were linked to the $\mathrm{Fe}$ abundance because the fit quality does not improve if they are treated as additional free parameters.

Table 2 shows the fluxes of the strongest RGS lines, measured with PINTofALE V2.0 (Kashyap \& Drake 2000), adopting a Lorentzian function to fit the line profile. We checked that the derived 3- $T$ EPIC model provides a reasonably good description of the RGS line fluxes.

We identified the OVII and Ne IX He-like triplets in the RGS spectra. The O VII lines (indicated as $r, i$, and $f$ in Fig. 2) provide a density-sensitive ratio $f / i=0.28 \pm 0.13$, which implies an electron density $\log N_{\mathrm{e}}=11.7 \pm 0.2$ for the plasma at $T \sim 2 \mathrm{MK}$. The Ne IX line flux measurements are affected by large uncertainties due to the strong blending with Fe lines. Figure 3 shows the observed Ne IX triplet with that predicted on the basis of the 3-T EPIC model assuming different $N_{\mathrm{e}}$ values.
Table 2. Strongest RGS lines of MP Mus.

\begin{tabular}{cclcr}
\hline \hline$\lambda_{\text {obs }}^{a}$ & $\lambda_{\text {pred }}^{a}$ & \multicolumn{1}{c}{ Ion } & $\log T_{\max }^{b}(F \pm \sigma F)^{c}$ \\
\hline 12.14 & 12.13 & Ne X Ne X Fe XVII & 6.80 & $22.2 \pm 3.4$ \\
12.30 & 12.28 & Fe XXI Fe XVII & 7.00 & $5.5 \pm 2.6$ \\
12.86 & 12.85 & Fe XX Fe XX Fe XX Fe XX & 7.00 & $3.4 \pm 2.3$ \\
13.46 & 13.45 & Ne IX Fe XIX & 6.60 & $21.1 \pm 3.7$ \\
13.55 & 13.52 & Ne IX Fe XIX & 6.90 & $8.6 \pm 3.1$ \\
13.73 & 13.70 & Ne IX & 6.60 & $8.8 \pm 2.8$ \\
14.19 & 14.21 & Fe XVIII Fe XVIII & 6.90 & $3.3 \pm 1.7$ \\
15.02 & 15.01 & Fe XVII & 6.70 & $11.8 \pm 2.9$ \\
15.21 & 15.18 & O VIII O VIII Fe XIX Fe XVII & 6.50 & $7.6 \pm 2.5$ \\
16.02 & 16.01 & O VIII O VIII Fe XVIII Fe XVIII & 6.50 & $22.6 \pm 6.3$ \\
16.78 & 16.78 & Fe XVII & 6.70 & $8.8 \pm 1.8$ \\
17.08 & 17.05 & Fe XVII Fe XVII & 6.70 & $17.2 \pm 3.0$ \\
18.65 & 18.63 & O VII & 6.30 & $8.7 \pm 2.6$ \\
18.98 & 18.97 & O VIII O VIII & 6.50 & $65.6 \pm 4.7$ \\
21.60 & 21.60 & O VII & 6.30 & $30.2 \pm 4.0$ \\
21.81 & 21.80 & O VII & 6.30 & $27.9 \pm 7.9$ \\
22.10 & 22.10 & O VII & 6.30 & $8.0 \pm 2.8$ \\
24.80 & 24.78 & N VII N VII & 6.30 & $10.5 \pm 2.8$ \\
28.49 & 28.47 & C VI C VI & 6.20 & $3.8 \pm 1.3$ \\
33.74 & 33.73 & C VI C VI & 6.10 & $16.4 \pm 5.2$ \\
\hline
\end{tabular}

$a$ Observed and predicted (APED database) wavelengths ( $)$. $b$ Temperature $(\mathrm{K})$ of maximum emissivity. ${ }^{c}$ Line fluxes $\left(10^{-6} \mathrm{ph} \mathrm{s}^{-1} \mathrm{~cm}^{-2}\right)$ with uncertainties at the $68 \%$ confidence level.

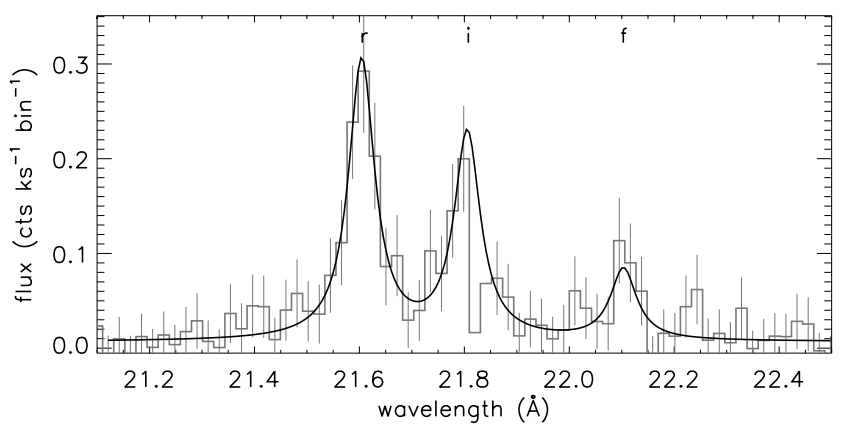

Fig. 2. RGS1 spectrum in the wavelength region of the O VII triplet (gray) with the best fit Lorentzian line profile (black). Wavelength bins corresponding to bad column pixels are plotted without error bars and were not considered in the fitting procedure.

This comparison suggests that the plasma at $T \sim 4 \mathrm{MK}$, which produces the Ne IX triplet, has a density $\log N_{\mathrm{e}}>11$, and possibly as high as that estimated from the O VII.

\section{Discussion}

From the analysis of the O VII triplet we find that the cool plasma component in our source has a density significantly higher than typical coronal values. It suggests that shock-heated plasma contributes significantly to the observed X-ray emission. In this respect, MP Mus is the fourth CTTS discovered so far with evidence of X-ray emission produced by cool high density plasma, likely resulting from an accretion process. Previous cases were TW Hya, BP Tau, and V4046 Sgr. Moreover, MP Mus also shows clear evidence of intense coronal activity, as indicated by the flares (see Fig. 1) and by the hot plasma component.

\subsection{Abundances}

The X-ray emitting plasma in MP Mus is heavily depleted of Fe, $\mathrm{Si}$, and $\mathrm{Mg} ; \mathrm{O}$, and $\mathrm{S}$ are moderately depleted, while $\mathrm{Ne}$ 


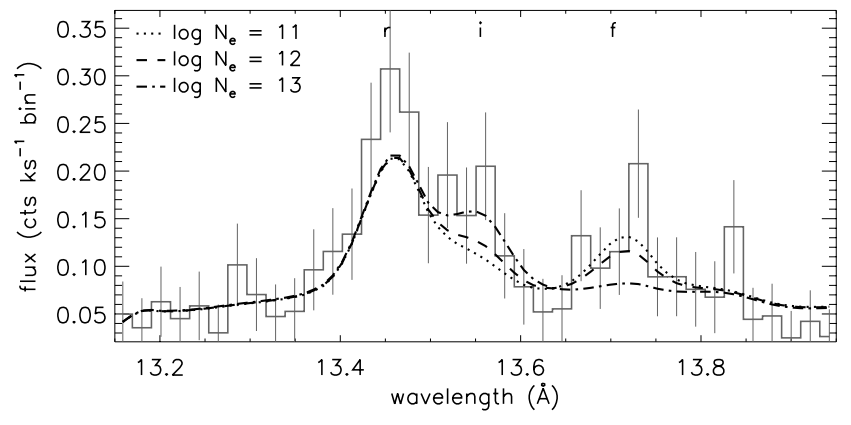

Fig. 3. RGS2 spectrum around the Ne IX triplet (gray) with the predicted spectra obtained from the 3-T EPIC model and assuming different electron densities.

Table 3. CTTS properties.

\begin{tabular}{lcrccc}
\hline \hline Star & $\begin{array}{c}\text { Stellar } \\
\text { Association }^{a}\end{array}$ & $\begin{array}{c}\text { Age } \\
(\mathrm{Myr})\end{array}$ & $\mathrm{Ne} / \mathrm{O}$ & $\begin{array}{c}T_{\text {med }}^{b} \\
(\mathrm{MK})\end{array}$ & $\begin{array}{c}N_{\mathrm{e}} \\
\left(10^{11} \mathrm{~cm}^{-3}\right)\end{array}$ \\
\hline BP Tau & Taurus & $0.6^{c}$ & $0.43^{d}$ & $16.1^{d}$ & $3.2^{d}$ \\
TW Hya & TWA & $8^{e}$ & $0.87^{f}$ & $4.8^{d}$ & $21.1^{d}$ \\
V4046 Sgr & BPMG & $12^{g}$ & $1.04^{h}$ & $6.4^{h}$ & $3.2^{h}$ \\
MP Mus & LCC & $17^{i}$ & 0.46 & 8.5 & 5.0 \\
\hline
\end{tabular}

$a$ TWA $=$ TW Hydrae association, BPMG $=\beta$ Pictoris Moving Group, LCC $=$ Lower Centaurus Curx; ${ }^{b} T_{\text {med }}$ is derived as $\left(\Sigma_{i} E M_{i} T_{i}\right) /\left(\Sigma_{i} E M_{i}\right)$; ${ }^{c}$ Gullbring et al. (1998); ${ }^{d}$ Robrade \& Schmitt (2006); ${ }^{e}$ Makarov \& Fabricius (2001); ${ }^{f}$ Drake et al. (2005); ${ }^{g}$ Zuckerman et al. (2001);

${ }^{h}$ Günther et al. (2006, $T_{\text {med }}$ has been derived from the line fluxes);

${ }^{i}$ Mamajek et al. (2002).

displays a larger abundance. If we assume that soft X-rays are produced by plasma heated in the accretion process, the observed abundances probe the chemical composition of the infalling circumstellar material. We compared the present abundance values of MP Mus with those obtained for the other three CTTSs showing evidence of X-ray emission due to shock-heated plasma (Table 3 ). In all cases the X-ray spectra indicate that the accreted material has a $\mathrm{Ne}$ abundance enhanced with respect to the other elements (with respect to the solar photospheric abundance ratios).

Stelzer \& Schmitt (2004) suggested that the non-solar abundances of the shock-heated plasma of TW Hya may be explained by assuming that the accreting material underwent grain depletion, a mechanism already invoked by Herczeg et al. (2002) to explain the low Si abundance. Depending on the temperature, the circumstellar material is composed of gas and dust phases which have different chemical compositions. The actual abundance ratios in the gas phase are determined by the different condensation temperatures $T_{\mathrm{c}}$ of the various elements (Savage \& Sembach 1996). One of the proposed scenarios of disk stratification predicts that dust grains mostly settle in the disk midplane, while the gas extends up to the disk surface, where it is easily ionized by the stellar radiation. Here it is funnelled along the magnetic field lines and accretes onto the central star. If the gas and dust phases are spatially separated in the inner circumstellar disk and/or the gas accretes more efficiently than the dust, the shock-heated material should be depleted of those elements (like Fe) which easily condense into dust grains and conversely should be enriched by more volatile elements (like noble gases). Following this scenario, the accreted material should have an abundance pattern similar to that observed in the interstellar gas, i.e. with the abundances decreasing for increasing $T_{\mathrm{c}}$. Note that the phenomenon of gas/dust separation in circumstellar disks, and the subsequent

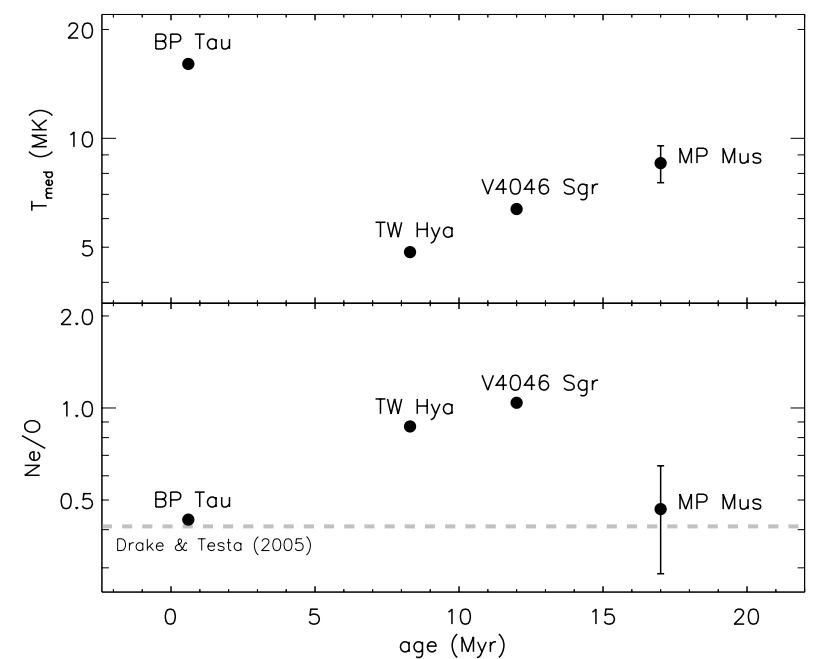

Fig. 4. Average plasma temperature and $\mathrm{Ne} / \mathrm{O}$ ratio vs. age for the sample of four CTTSs with evidence of high density cool plasma.

accretion of only the gas phase, is also considered responsible for the peculiar abundances of RV Tauri stars (see Giridhar et al. 2005, and references therein).

Drake et al. (2005) showed that TW Hya displays a Ne/O ratio larger by a factor $\sim 2$ than the uniform value observed in a large sample of coronal X-ray sources (Drake \& Testa 2005). Moreover, $\mathrm{Ne} / \mathrm{Fe}$ abundance ratios as large as that of TW Hya were observed only in a very few cases of putatively coronal sources. These results support the hypothesis that soft X-ray radiation from TW Hya is not produced by coronal plasma. Drake et al. suggest that the $\mathrm{Ne} / \mathrm{O}$ ratio can be used as a criterion to identify X-ray sources where the emitting material in the accretion stream suffered grain depletion. However, we note that the condensation temperature of $\mathrm{O}$ is quite low $(180 \mathrm{~K})$, therefore the separation between gas and dust must occur at low temperature to produce significant $\mathrm{O}$ depletion in the accretion streams.

A large $\mathrm{Ne} / \mathrm{O}$ abundance ratio is also observed in the X-ray spectrum of V4046 Sgr (Günther et al. 2006), where the high density again hints at X-rays from shock-heated plasma. Instead, both MP Mus and BP Tau have Ne/O ratios typical of stellar coronae. Drake et al. (2005) explained the Ne/O ratio of BP Tau, lower than that of TW Hya, on the basis of the different evolutionary stages of their circumstellar disks. Since TW Hya is significantly older than BP Tau, it is conceivable that the dust/gas separation process, and the subsequent depletion of high $T_{\mathrm{c}}$ elements, is not visible in the latter case because these processes occur on a time scale longer than the age of BP Tau ( 0.6 Myr).

In Table 3 we report the ages of the four CTTSs introduced above. We adopt an age of 17 Myr for MP Mus (Mamajek et al. 2002), since it is compatible with the age of the LCC association. For the subsequent discussion, the absolute age of each CTTS is unimportant, while only the age sequence matters, whose reliability depends only on the correctness of the membership of these CTTSs to the relevant stellar associations.

Figure 4 shows the variations of plasma average temperature and $\mathrm{Ne} / \mathrm{O}$ ratio with respect to stellar age, for the sample of four CTTSs (having spectral types ranging from K1 to K7). Both $T_{\text {med }}$ and $\mathrm{Ne} / \mathrm{O}$ do not have a monotonic trend with age, but these two plots suggest that stars with hotter plasma have lower Ne/O ratios, and vice versa. It is likely that high $T_{\text {med }}$ indicates a large contribution from coronal plasma to the whole $\mathrm{X}$-ray emission. In this scenario of mixed accretion-driven and coronal $\mathrm{X}$-ray emission, the measured $\mathrm{Ne} / \mathrm{O}$ ratio is a weighted 
average of the values in the shock-heated plasma and in the coronal plasma. Hence any large $\mathrm{Ne} / \mathrm{O}$ ratio of the accreted material may be partly hidden by the coronal plasma abundances. To check this possibility we fitted the observed EPIC spectra of MP Mus assuming a high Ne/O ratio for the coolest plasma component, but the model does not reproduce the observed spectra as well as the model described in Sect. 2. Moreover, in MP Mus the hot coronal plasma does not contribute significantly to the observed $\mathrm{O}$ and $\mathrm{Ne}$ line emission (see below).

We conclude that the relatively low Ne/O ratio in MP Mus is a characteristic of the cool accretion component, and the stellar age is likely not the only parameter that determines the $\mathrm{Ne} / \mathrm{O}$ ratio observed in CTTSs with evidence of high density cool plasma.

\subsection{Accretion}

For the following discussion we first assume that the cool X-ray emitting plasma of MP Mus is only due to the shock accretion, with no contribution from coronal plasma. Starting from the Mamajek et al. (2002) results on MP Mus, and based on the Siess et al. (2000) stellar models, we adopt for MP Mus a mass of $1.2 M_{\odot}$ and a radius of $1.3 R_{\odot}$.

Using the O VII triplet and the O VIII Ly $\alpha$ lines we infer the electron density $\left(N_{\mathrm{e}}=5 \times 10^{11} \mathrm{~cm}^{-3}\right)$, temperature $(T=3 \mathrm{MK}$, obtained from the O VIII Ly $\alpha$ and O VII $r$ lines), and emission measure $\left(E M=2.4 \times 10^{53} \mathrm{~cm}^{-3}\right)$ of the post shock plasma. In the strong shock scenario, the relevant plasma parameters are linked by the relations:

$N_{1}=4 N_{0}, \quad v_{1}=\frac{1}{4} v_{0}, \quad T_{1}=\frac{3}{16} \frac{\mu m_{\mathrm{H}}}{k} v_{0}^{2}$

where the suffixes 0 and 1 indicate the pre-shock and post-shock plasma, $N$ the density, $v$ the velocity, $T$ the temperature, and $\mu$ the mean molecular weight (in our case $\mu=0.61$ ). From the measured temperature $T_{1}$ we infer that the pre-shock velocity is $470 \mathrm{~km} \mathrm{~s}^{-1}$. This value corresponds to a free fall from an inner radius of the circumstellar disk of $3 R_{\star}$, or from a larger distance if some energy loss occurs during the fall. From the post-shock plasma temperature and density we derive a cooling time of $350 \mathrm{~s}$, and considering that the post-shock velocity is $120 \mathrm{~km} \mathrm{~s}^{-1}$, we obtain a characteristic length of the post-shock region $l=4 \times 10^{9} \mathrm{~cm}=0.05 R_{\star}$. Hence, the cross section of the infalling stream $A=E M /\left(N_{\mathrm{e}} N_{\mathrm{H}} l\right)$ is $3 \times 10^{20} \mathrm{~cm}^{2}$. It corresponds to a filling factor $f=A /\left(4 \pi R_{\star}^{2}\right)$ of $0.3 \%$ of the stellar surface, and to a mass accretion rate of $5 \times 10^{-11} M_{\odot} \mathrm{yr}^{-1}$.

We made the hypotheses that: (1) the cool plasma is produced in the accretion shock; (2) the cool plasma is optically thin; (3) its density is measured from the O VII $f / i$.

We are confident that the assumption (1) is appropriate. The two strong flares detected, produced by coronal plasma, contribute just $3.6 \%$ of the spectrum above $18 \AA$ (i.e. below $0.7 \mathrm{keV}$ ); also, we tried to fit the O VII triplet with two contributions due to low and high electron density $\left(10^{9}\right.$ and $10^{12} \mathrm{~cm}^{-3}$, respectively), finding that at least $80 \%$ of the $\mathrm{O}$ VII is due to high density (i.e. shock-heated) plasma, and at most $20 \%$ to low density (i.e. coronal) plasma.

Hence the derived accretion rate $\dot{M}$, which depends only on hypotheses (1) and (2), but not on $N_{\mathrm{e}}$, is acceptable, but a larger $\dot{M}$ could be possible if part of the X-ray emission is absorbed.

The measured $N_{\mathrm{e}}$ is more uncertain: a small contribution of low $N_{\mathrm{e}}$ coronal plasma to the O VII triplet might cause an underestimation of $N_{\mathrm{e}}$; conversely an UV field might influence the populations of the $\mathrm{O}$ VII atomic levels by photoexcitation and hence mimic a high density plasma. No UV excess emission, which could originate from the accretion hot spot, has been reported for this star. However a sufficiently high UV radiation density can be present only very near the accretion hot spot on the stellar surface, and the photoexcitation hypothesis would anyway indicate that the cool X-ray emitting plasma is close to the base of the accretion funnel.

\section{Conclusions}

From the analysis of the XMM-Newton observation of the CTTS MP Mus we derived evidence that plasma heated in the accretion shock produces the soft part of the X-ray emission. We measured a Ne/O ratio similar to that of BP Tau and reduced by a factor of 2 with respect to that of TW Hya and V4046 Sgr: this result suggests that the stellar age is not a useful parameter to predict the amount of grain depletion suffered by the accreting material.

Acknowledgements. C.A., A.M., and G.P. acknowledge partial support for this work from contract ASI-INAF I/023/05/0 and from the Ministero dell'Università e della Ricerca. Based on observations obtained with XMM-Newton, an ESA science mission with instruments and contributions directly funded by ESA Member States and NASA.

\section{References}

Arnaud, K. A. 1996, in Astronomical Data Analysis Software and Systems V, ed. G. H. Jacoby, \& J. Barnes, ASP Conf. Ser., 101, 17

Asplund, M., Grevesse, N., \& Sauval, A. J. 2005, in Cosmic Abundances as Records of Stellar Evolution and Nucleosynthesis, ed. T. G. Barnes, III, \& F. N. Bash, ASP Conf. Ser., 336, 25

Batalha, C. C., Quast, G. R., Torres, C. A. O., et al. 1998, A\&AS, 128, 561

Calvet, N., \& Gullbring, E. 1998, ApJ, 509, 802

Carpenter, J. M., Wolf, S., Schreyer, K., Launhardt, R., \& Henning, T. 2005, AJ, 129,1049

Drake, J. J., \& Testa, P. 2005, Nature, 436, 525

Drake, J. J., Testa, P., \& Hartmann, L. 2005, ApJ, 627, L149

Feigelson, E. D., \& Montmerle, T. 1999, ARA\&A, 37, 363

Giridhar, S., Lambert, D. L., Reddy, B. E., Gonzalez, G., \& Yong, D. 2005, ApJ, 627,432

Gregorio-Hetem, J., Lepine, J. R. D., Quast, G. R., Torres, C. A. O., \& de La Reza, R. 1992, AJ, 103, 549

Güdel, M., Skinner, S. L., Mel'nikov, S. Y., et al. 2007, A\&A, in press [arXiv: astro-ph/0612589]

Gullbring, E., Hartmann, L., Briceno, C., \& Calvet, N. 1998, ApJ, 492, 323

Günther, H. M., Liefke, C., Schmitt, J. H. M. M., Robrade, J., \& Ness, J. 2006, A\&A, 459, L29

Herczeg, G. J., Linsky, J. L., Valenti, J. A., Johns-Krull, C. M., \& Wood, B. E. 2002, ApJ, 572, 310

Kashyap, V., \& Drake, J. J. 2000, Bull. Astron. Soc. India, 28, 475

Kastner, J. H., Huenemoerder, D. P., Schulz, N. S., Canizares, C. R., \& Weintraub, D. A. 2002, ApJ, 567, 434

Makarov, V. V., \& Fabricius, C. 2001, A\&A, 368, 866

Mamajek, E. E., Meyer, M. R., \& Liebert, J. 2002, AJ, 124, 1670

Ness, J.-U., Güdel, M., Schmitt, J. H. M. M., Audard, M., \& Telleschi, A. 2004, A\&A, 427, 667

Preibisch, T., Kim, Y.-C., Favata, F., et al. 2005, ApJS, 160, 401

Robrade, J., \& Schmitt, J. H. M. M. 2006, A\&A, 449, 737

Sartori, M. J., Lépine, J. R. D., \& Dias, W. S. 2003, A\&A, 404, 913

Savage, B. D., \& Sembach, K. R. 1996, ARA\&A, 34, 279

Schmitt, J. H. M. M., Robrade, J., Ness, J.-U., Favata, F., \& Stelzer, B. 2005, A\&A, 432, L35

Siess, L., Dufour, E., \& Forestini, M. 2000, A\&A, 358, 593

Silverstone, M. D., Meyer, M. R., Mamajek, E. E., et al. 2006, ApJ, 639, 1138

Smith, R. K., Brickhouse, N. S., Liedahl, D. A., \& Raymond, J. C. 2001, ApJ, 556, L91

Stelzer, B., \& Schmitt, J. H. M. M. 2004, A\&A, 418, 687

Telleschi, A., Güdel, M., Briggs, K. R., et al. 2007, A\&A, in press [arXiv: astro-ph/0610456]

Testa, P., Drake, J. J., \& Peres, G. 2004, ApJ, 617, 508

Zuckerman, B., Song, I., Bessell, M. S., \& Webb, R. A. 2001, ApJ, 562, L87 